УДК 621.315 .592

\title{
Влияние термической предыстории на свойства эффективных термоэлектрических сплавов $\mathrm{Ge}_{0.86} \mathrm{~Pb}_{0.1} \mathrm{Bi}_{0.04} \mathrm{Te}^{*}$
}

\author{
(C) А.А. Шабалдин ${ }^{1}$, А.Ю. Самунин ${ }^{1}$, П.П. Константинов ${ }^{1}$, С.В. Новиков ${ }^{1}$, \\ A.T. Бурков ${ }^{1, \pi}$, Zhonglin $\mathrm{Bu}^{2}$, Yanzhong Pei ${ }^{2}$ \\ ${ }^{1}$ Физико-технический институт им. А.Ф. Иоффе Российской академии наук, \\ 194021 Санкт-Петербург, Россия \\ ${ }^{2}$ Interdisciplinary Materials Research Center, School of Materials Science and Engineering, \\ Tongji University, Shanghai, China \\ ^ E-mail: berrior@rambler.ru
}

Поступила в Редакцию 20 ноября 2021 г.

В окончательной редакции 25 ноября 2021 г.

Принята к публикации 25 ноября 2021 г.

\begin{abstract}
Изучаются свойства сплавов на основе соединения GeTe с частичным замещением германия свинцом и легированием висмутом: $\mathrm{Ge}_{0.86} \mathrm{~Pb}_{0.1} \mathrm{Bi}_{0.04} \mathrm{Te}$. Цель исследования - изучение возможности повышения термоэлектрической эффективности соединения путем комбинации оптимального легирования и изовалентного замещения для улучшения электронных свойств с одновременным снижением решеточной теплопроводности. Изучены образцы сплавов, изготовленные в двух разных исследовательских лабораториях по похожим, но не полностью идентичным процедурам. Показано, что электронные (термоэдс и электропроводность) свойства образцов двух групп хорошо согласуются друг с другом. При этом свойства сплавов зависят от термической предыстории образцов из-за наличия при температурах $600-800 \mathrm{~K}$ фазового перехода из низкотемпературной ромбоэдрической в высокотемпературную кубическую структурную модификацию. Параметр термоэлектрической эффективности сплавов достигает максимального значения 1.5 при температуре $\sim 750 \mathrm{~K}$.
\end{abstract}

Ключевые слова: термоэлектрический сплав, термоэдс, электропроводность, теплопроводность.

DOI: 10.21883/FTP.2022.03.52107.34

\section{1. Введение}

Теллуриды свинца, олова и германия являются основой самых эффективных современных среднетемпературных термоэлектрических материалов [1]. Эти материалы используются в термоэлектрических генераторах для наземных и космических применений. Несмотря на долгую историю исследований и применений этих соединений, их интенсивное изучение с целью повышения термоэлектрической эффективности и улучшения механических свойств продолжается, особенно в последние годы. Эти усилия привели, как следует из публикаций, к значительным успехам. Параметр термоэлектрической эффективности $Z T=\frac{S^{2} \sigma}{\kappa} T$ (здесь $S$ - коэффициент термоэдс, $\sigma$ - электропроводность, $\kappa-$ теплопроводность, $T-$ абсолютная температура) [2-4], согласно литературным данным, достигает значений, значительно превышающих 2 [5-13]. Однако, хотя публикации с высокими значениями параметра $Z T$ в соединениях на основе $\mathrm{PbTe}, \mathrm{GeTe}$ появились около 10 лет назад $[14,15]$, сведений об их практическом применении до сих пор нет. Одной из причин подобного положения является плохая воспроизводимость рекордных результатов. Это обстоятельство может быть связано как с высокой чувствитель-

* Доклад на XXVII Межгосударственной конференции „Термоэлектрики и их применения“" (ISCTA 2021), Санкт-Петербург, $13-16$ сентября 2021 г. ностью термоэлектрических свойств соединений к параметрам технологических процессов при их получении, так и с субъективными факторами. Во многих случаях высокие значения ZT получаются благодаря очень низкой решеточной теплопроводности в сплавах и сильно легированных соединениях из-за рассеяния фононов на точечных дефектах, а также на дефектах структуры: границах кристаллических зерен, зародышах примесных фаз и дислокациях. Кроме того, в сплавах на основе GeTe в силу присущей этому соединению структурной неустойчивости возможно возникновение мягких фононных мод, приводящих к усилению фононфононного рассеяния. Субъективным фактором может быть отсутствие принятых стандартов для измерения термоэлектрических параметров и связанная с этим значительная неопределенность при их измерении в разных исследовательских группах. В особенности, это относится к измерениям теплопроводности. Термоэлектрические материалы, как правило, имеют очень низкую теплопроводность, и ее измерение требует хорошего понимания соответствующих процедур и строгого их соблюдения. Сейчас подавляющее число исследовательских групп использует нестационарные методы измерения температуропроводности $(\alpha)$, по которой затем определяется теплопроводность материала: $\kappa=\alpha C_{p} d$, где $C_{p}-$ теплоемкость при постоянном давлении, a $d-$ плотность. При этом для определения двух других транспортных коэффициентов $(S$ и $\sigma)$, опреде- 
ляющих вместе с $\kappa$ параметр термоэлектрической эффективности $Z T$, применяется отдельная измерительная установка и, как правило, другой образец. Другой метод определения параметра ZT основан на использовании стационарной процедуры измерения теплопроводности, которая одновременно позволяет измерить коэффициент термоэдс и коэффициент электропроводности и определить параметр $Z T$ с использованием только одного образца $[16,17]$.

В этой работе мы изучаем термоэлектрические свойства сплавов GeTe и $\mathrm{PbTe}$, легированные висмутом: $\mathrm{Ge}_{0.86} \mathrm{~Pb}_{0.1} \mathrm{Bi}_{0.04} \mathrm{Te}$. Соединение GeTе из-за большой концентрации равновесных вакансий $\mathrm{Ge}$ является полупроводником $p$-типа с высокой для термоэлектрических применений концентрацией дырок [18]. Для снижения концентрации применяется легирование элементами 5 группы, обычно висмутом или сурьмой. При этом было показано, что при частичном изовалентном замещении $\mathrm{Ge}$ на $\mathrm{Pb}$ эффективность легирования висмутом возрастает $[19,20]$. Кроме того, изовалентное замещение свинцом понижает решеточную теплопроводность, не приводя к пропорциональному снижению подвижности носителей заряда. В результате в сплавах $\mathrm{Ge}_{0.91} \mathrm{~Pb}_{0.05} \mathrm{Bi}_{0.04} \mathrm{Te}$ параметр $Z T$ достигает 1.5 при температурах 670-800 K [19]. Мы изучаем возможность дальнейшего повышения эффективности в сплавах с увеличенным содержанием $\mathrm{Pb}$. Второй задачей этой работы является изучение влияния термической предыстории на термоэлектрические свойства сплавов. GeTe существует в двух модификациях: низкотемпературной ромбоэдрической $(R 3 m)$ и высокотемпературной кубической $(F m \overline{3} m)$. В чистом GeTe температура перехода составляет $703 \mathrm{~K}$ [21], в сплавах температура перехода зависит от состава, уровня и типа легирования [19]. Свойства GeTe и сплавов на его основе зависят от фазового состояния материала, которое в свою очередь может сложным образом зависеть от термической истории образца: температуры отжига, темпа охлаждения и нагрева и т.д. Поэтому для правильного определения термоэлектрических свойств сплавов необходимо понимание связи между структурным состоянием материала и его термической историей. И наконец, мы выполняем сравнительное исследование термоэлектрических свойств образцов одинакового состава, но изготовленных в двух разных лабораториях: в лаборатории физики термоэлементов ФТИ (далее образцы FTI) и в лаборатории профессора Пеи (Y. Реi) в университете Тонгжи (Tongji University), Шанхай, КНР (далее образцы Tongji). Недавно в этой группе был исследован сплав $\mathrm{Ge}_{0.86} \mathrm{~Pb}_{0.1} \mathrm{Bi}_{0.04} \mathrm{Te}$ и получено рекордное значение параметра $Z T=2.4$ [9]. Нашей задачей является проверка воспроизводимости таких высоких параметров эффективности.

\section{2. Образцы и методика эксперимента}

Поликристаллические

$\mathrm{Ge}_{0.86} \mathrm{~Pb}_{0.1} \mathrm{Bi}_{0.04} \mathrm{Te}$ были сплавлением компонентов: $\mathrm{Ge}(99.999 \%), \mathrm{Pb}(99.99 \%)$, $\mathrm{Bi}(99.99 \%)$ Te $(99.96 \%)$ в откачанной кварцевой ампуле при $T=1123 \mathrm{~K}$. Синтезированные слитки отжигались в течение 120 ч при температуре $773 \mathrm{~K}$ с целью гомогенизации. Стандартный рентгенофазовый анализ подтвердил ромбоэдрическую структуру с параметрами $a=4.217 \pm 0.002 \AA, c=10.684 \pm 0.006 \AA$ (в гексагональном представлении), в разумном соответствии с литературными данными [22-24]. Обнаружено также присутствие $~ 1 \%$ кубической фазы.

Отожженный слиток был измельчен и просеян через сито с размером ячейки 0.25 мм. Из порошка методом горячего прессования при температуре $653 \mathrm{~K}$ и давлении 500 МПа были приготовлены образцы для измерения термоэлектрических свойств в виде параллелепипедов $9 \times 9 \times 15$ мм и в виде пластинок $9 \times 2 \times 15$ мм. Образцы дополнительно отжигались при температуре $770 \mathrm{~K}$ в течение 100 ч и затем охлаждались в печи.

Образцы в группе проф. Пеи были изготовлены по похожей технологии с некоторым отличием в параметpax: гомогенизирующий отжиг проводился при более высокой температуре $(923 \mathrm{~K})$ в течение 72 ч, а горячее прессование производилось при температуре $873 \mathrm{~K}$ при давлении $80 \mathrm{MПа} \mathrm{в} \mathrm{течение} 40$ мин. Образцы для измерения термоэлектрических свойств были изготовлены в виде параллелепипедов $6 \times 6 \times 10$ мм.

Измерение термоэлектрических свойств проводилось в ФТИ с использованием двух оригинальных экспериментальных установок. Коэффициент термоэдс, электропроводность и теплопроводность измерялись одновременно в диапазоне температур 300-800 K. Для измерения теплопроводности использовался стационарный классический метод [16]. Электропроводность измерялась 4-зондовым методом на постоянном токе, а термоэдс - дифференциальным методом. Погрешность определения параметра $Z T$ по результатам измерений на этой установке не превышает 10\%. Для in situ измерений коэффициента термоэдс и электропроводности при термоциклировании и отжиге использовалась установка, позволяющая выполнять измерения в динамическом температурном режиме, скорость изменения температуры при этих измерения находилась в диапазоне $0-10 \mathrm{~K} /$ мин $[25,26]$.

\section{3. Экспериментальные результаты и обсуждение}

Результаты измерения термоэлектрических свойств образцов сплавов $\mathrm{Ge}_{0.86} \mathrm{~Pb}_{0.1} \mathrm{Bi}_{0.04} \mathrm{Te}$, изготовленных в ФТИ (обозначены как FTI) и в университете Тонгжи (Tongji), представлены на рис. 1 вместе с литературными данными [9]. В целом, учитывая различия в технологии 

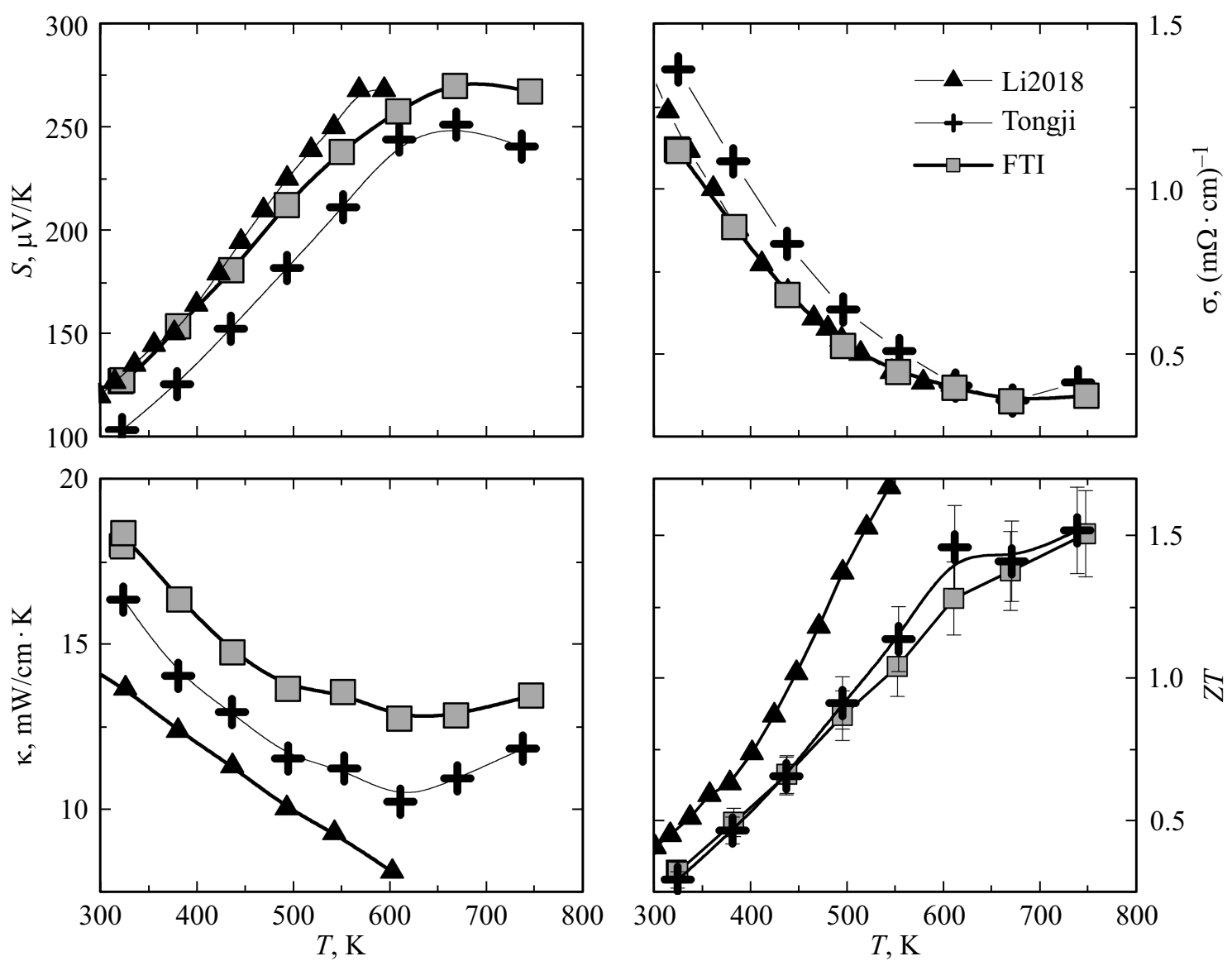

Рис. 1. Температурные зависимости термоэдс, электропроводности, теплопроводности и безразмерного параметра термоэлектрической эффективности сплавов $\mathrm{Ge}_{0.86} \mathrm{~Pb}_{0.1} \mathrm{Bi}_{0.04}$ Те. Для сравнения показаны также результаты работы [9] (Li2018).

приготовления образцов, результаты неплохо согласуются друг с другом. Коэффициент термоэдс образца FTI больше, чем у образца Tongji, но у последнего при этом более высокая электропроводность. Наиболее заметные различия наблюдаются в теплопроводности. Несмотря на немного меньшую электропроводность, теплопроводность образца FTI заметно больше теплопроводности образца группы Tongji. Однако параметр термоэлектрической эффективности, измеренный в ФТИ, у обоих образцов практически совпадает, и при этом он значительно уступает литературным данным, в основном из-за значительно более низкой теплопроводности, полученной в работе [9].

Дальнейшее, более детальное исследование показало, что электронные свойства (коэффициент термоэдс и электропроводность) образцов Tongji и FTI также практически идентичны. Различия, которые видны на рис. 1 , в основном связаны с различной термической предысторией образцов. Это иллюстрирует рис. 2, на котором показаны температурные зависимости термоэдс и электропроводности образца Tongji, измеренные при нагреве до $800 \mathrm{~K}$ и последующем охлаждении. Эти измерения были выполнены после измерений, представленных на рис. 1. Температурные зависимости обоих свойств этого образца при повторном нагреве совпадают с соответствующими зависимостями образца FTI, измеренными на предыдущем этапе (рис. 1), а зависимости,

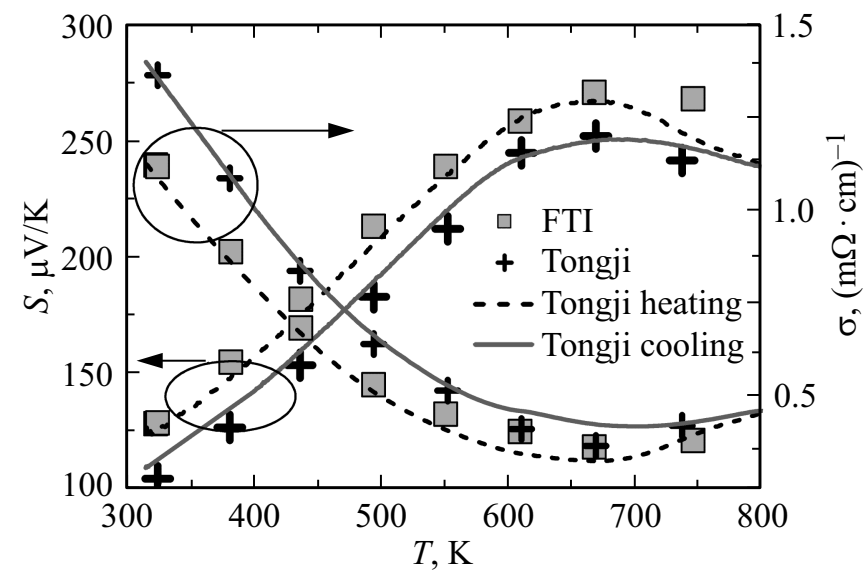

Рис. 2. Температурные зависимости коэффициента термоэдс и электропроводности образцов сплава $\mathrm{Ge}_{0.86} \mathrm{~Pb}_{0.1} \mathrm{Bi}_{0.04} \mathrm{Te}$. Символы представляют результаты измерений в стационарных условиях при нагреве образцов FTI и Tongji, показанные на рис. 1, а линии - последующие измерения образца Tongji при нагреве и охлаждении со скоростью $\sim 5 \mathrm{~K} /$ мин. 
измеренные при охлаждении, совпадают с зависимостями для этого же образца (Tongji), измеренными также на предыдущем этапе (рис. 1). Таким образом, электронные свойства образцов обеих групп совпадают в пределах погрешности измерений при учете термической предыстории этих образцов.

Важно отметить, что сплав после перехода в высокотемпературную фазу, при последующем охлаждении со скоростью $\sim 5 \mathrm{~K} /$ мин не возвращается в исходное низкотемпературное состояние. Для того чтобы произошел обратный переход в низкотемпературную фазу требуется длительный отжиг при достаточно высокой температуре. Образец FTI перед измерениями, результаты которых показаны на рис. 1, отжигался при температуре $770 \mathrm{~K}$, и затем медленно (в течение $\sim 24$ ч) охлаждался в печи. Во время этого медленного охлаждения происходит переход в низкотемпературную структурную модификацию. Поэтому результаты, полученные при измерении этого образца при его нагреве (рис. 1), характеризуют свойства низкотемпературной фазы сплава $\mathrm{Ge}_{0.86} \mathrm{~Pb}_{0.1} \mathrm{Bi}_{0.04}$ Те. Образец Tongji был быстро охлажден после горячего прессования, и переход в низкотемпературную фазу в нем не произошел. Поэтому результаты измерений этого образца при первом нагреве (рис. 1) характеризуют свойства метастабильной высокотемпературной модификации. Последующее охлаждение в установке для стационарных измерений происходит медленно, так температура от $750 \mathrm{~K}$ снижается до $500 \mathrm{~K}$ за $\sim 3-4$ ч. За это время происходит обратный переход в низкотемпературную структурную фазу. Поэтому результаты измерения при следующем нагреве этого образца (рис. 2) отражают свойства низкотемпературной модификации и совпадают с результатами для образца FTI.

Рис. 3 показывает эволюцию свойств образца Tongji этого сплава в процессе нагрева-охлаждения и отжига при температуре, ниже температуры фазового превращения в высокотемпературную кубическую модификацию. Образец перед этим измерением находился в метастабильной высокотемпературной модификации в результате первого цикла нагрев-охлаждение, показанного на рис. 2. Образец был снова нагрет до $600 \mathrm{~K}$ и при этой температуре отожжен in situ в течение 10 ч. В результате отжига происходит фазовый переход в низкотемпературную структурную модификацию, коэффициент термоэдс (при $600 \mathrm{~K}$ ) увеличился при этом на $\sim 8 \%$, а электропроводность уменьшилась более чем на $20 \%$. Свойства сплава при термоциклировании полностью воспроизводимы при правильном выборе режимов термообработки: при термоциклировании с относительно быстрым темпом нагрева и охлаждения образец после нагрева до $\sim 800 \mathrm{~K}$ будет все время находиться в высокотемпературной модификации; при термоциклировании с промежуточным отжигом (после нагрева до $800 \mathrm{~K}$ ) при температуре 600-650 К будет происходить возврат в низкотемпературную фазу с соответствующими изменениями свойств. Характерное время перехода из высоко-

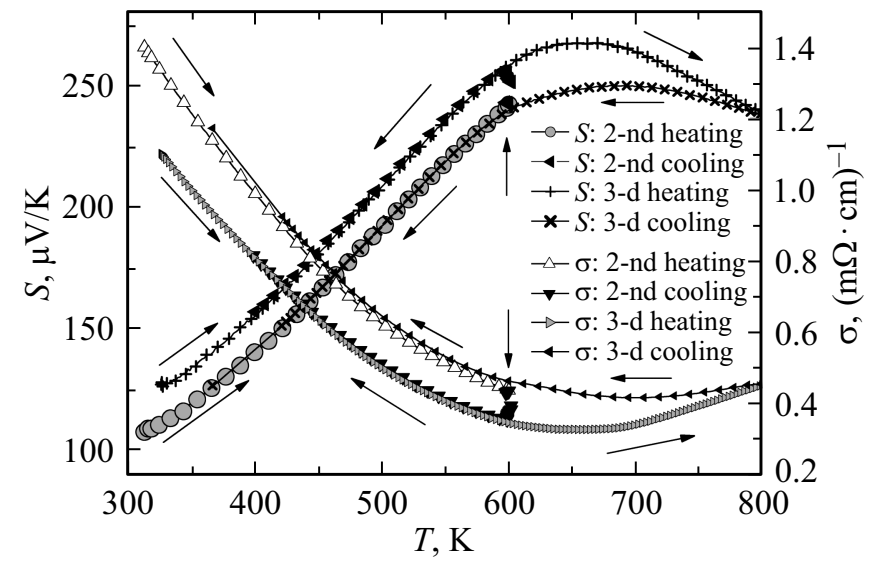

Pис. 3. Температурные зависимости коэффициента термоэдс и электропроводности образца Tongji сплава $\mathrm{Ge}_{0.86} \mathrm{~Pb}_{0.1} \mathrm{Bi}_{0.04} \mathrm{Te}$. Образец был нагрет от комнатной температуры до $600 \mathrm{~K}$ и отожжен при этой температуре in situ в течение 10 ч, после чего охлажден до комнатной температуры. Затем был повторен цикл с нагревом до $800 \mathrm{~K}$ и охлаждением до комнатной температуры. Стрелки показывают направление изменения температуры.

температурной модификации сплава $\mathrm{Ge}_{0.86} \mathrm{~Pb}_{0.1} \mathrm{Bi}_{0.04} \mathrm{Te}$ в низкотемпературную при температуре $600 \mathrm{~K}$ составляет несколько часов. Очевидно, что это время сильно зависит от температуры отжига.

\section{4. Заключение}

Термоэлектрические свойства образцов сплавов $\mathrm{Ge}_{0.86} \mathrm{~Pb}_{0.1} \mathrm{Bi}_{0.04} \mathrm{Te}$, изготовленных в двух разных лабораториях по близким, но не идентичным технологиям, и измеренные с использованием оборудования одной из лабораторий (ФТИ), в целом совпадают. Это указывает на хорошую воспроизводимость свойств этих сплавов и устойчивость по отношению к небольшим изменениям процедур синтеза материалов и изготовления образцов.

Термоэлектрические свойства сплавов зависят от термической предыстории образца из-за наличия фазового перехода между двумя структурными модификациями в интервале температур 600-800 К. Высокотемпературная кубическая модификация при охлаждении образцов со скоростью $\sim 1 \mathrm{~K} /$ Мин и выше сохраняется при низких температурах длительное время. При температуре $600 \mathrm{~K}$ фазовый переход из высокотемпературной в низкотемпературную модификацию завершается при отжиге за время $\sim 5$ ч.

Параметр термоэлектрической эффективности $Z T$ сплавов $\mathrm{Ge}_{0.86} \mathrm{~Pb}_{0.1} \mathrm{Bi}_{0.04} \mathrm{Te}$ близок к параметру сплавов $\mathrm{Ge}_{0.91} \mathrm{~Pb}_{0.05} \mathrm{Bi}_{0.04} \mathrm{Te}$ [19]. Максимальное значение $Z T$ сплава $\mathrm{Ge}_{0.91} \mathrm{~Pb}_{0.05} \mathrm{Bi}_{0.04} \mathrm{Te}$ немного выше, чем у сплава $\mathrm{Ge}_{0.86} \mathrm{~Pb}_{0.1} \mathrm{Bi}_{0.04} \mathrm{Te}$, однако средние по интервалу температур $320-740 \mathrm{~K}$ значения ZT совпадают и составляют 0.97. Поэтому с точки зрения перспек- 
тив практического применения сплав $\mathrm{Ge}_{0.86} \mathrm{~Pb}_{0.1} \mathrm{Bi}_{0.04} \mathrm{Te}$, возможно, окажется предпочтительным. Этот вопрос, однако, требует дополнительных исследований, в частности механических свойств и термической стабильности сплавов.

В данной работе мы не изучали влияние термической предыстории на теплопроводность сплавов из-за трудоемкости таких исследований и недостатка образцов необходимого размера. Такое исследование, очевидно, необходимо и планируется в ближайшем будущем.

\section{Финансирование работы}

Работа поддержана Российским фондом фундаментальных исследований, грант № 18-52-80005 (БРИКС) и Национальным фондом естественных наук Китая (National Natural Science Foundation of China), грант 51861145305 (БРИКС проект).

\section{Конфликт интересов}

Авторы заявляют, что у них нет конфликта интересов.

\section{Список литературы}

[1] Ю.И. Равич, Б.А. Ефимова, И.А. Смирнов. Методы исследования полупроводников в применении к халькогенидам свинца PbTe, PbSe и $\mathrm{PbS}$ (М., Наука, 1968).

[2] А.Ф. Иоффе. Энергетические основы термоэлектрических батарей на полупроводниках (М., Изд-во AH CCCP, 1950).

[3] А.Ф. Иоффе. ЖТФ, 23, 1452 (1953).

[4] А.Ф. Иоффе. Физика полупроводников (М., Изд-во AH CCCP, 1957).

[5] Y. Gelbshtein, J. Davidow. Phys. Chem. Chem. Phys., 16, 20120 (2014).

[6] J. Li, Z. Chen, X. Zhang, H. Yu, Z. Wu, H. Xie, Y. Chen, Y. Pei. Adv. Sci., 4, 1700341 (2017).

[7] J. Li, X. Zhang, X. Wang, Z. Bu, L. Zheng, B. Zhou, F. Xiong, Y. Chen, Y. Pei. J. Am. Chem. Soc., 140 (47), 16190 (2018).

[8] M. Hong, Z.-G. Chen, L. Yang, Y.-C. Zou, M.S. Dargusch, H. Wang, J. Zou. Adv. Mater., 30, 1705942 (2018).

[9] J. Li, X. Zhang, Z. Chen, S. Lin, W. Li, J. Shen, I. T. Witting, A. Faghaninia, Y. Chen, A. Jain, L. Chen, G.J. Snyder, Y. Pei. Joule, 2, 976 (2018).

[10] X. Zhang, J. Li, X. Wang, Z. Chen, J. Mao, Y. Chen, Y. Pei. J. Am. Chem. Soc., 140, 15883 (2018).

[11] R.K. Vankayala, T.-W. Lan, P. Parajuli, F. Liu, R. Rao, S.H. Yu, T.-L. Hung, C.-H. Lee, S. Yano, C.-R. Hsing, D.-L. Nguyen, C.L. Chen, S. Bhattacharya, K.-H. Chen, M.-N. Ou, O. Rancu, A.M. Rao, Y.-Y. Chen. Adv. Sci., 7 (24), 2002494 (2020).

[12] T. Parashchuk, B. Wiendlocha, O. Cherniushok, R. Knura, K.T. Wojciechowski. ACS Appl. Mater. Interfaces, 13, 49027 (2021).

[13] Y.-F. Tsai, P.P.-C. Wei, L. Chang, K.-K. Wang, C.-C. Yang, Y.-C. Lai, C.-R. Hsing, C.-M. Wei, J. He, G.J. Snyder. Adv. Mater., 33, 2005612 (2021).

[14] M.G. Kanatzidis. Chem. Mater., 22, 648 (2010).
[15] K. Biswas, J. He, I.D. Blum, C.I. Wu, T.P. Hogan, D.N. Seidman, V.P. Dravid, M.G. Kanatzidis. Nature, 489, 414 (2012).

[16] А.В. Петров. Термоэлектрические свойства полупроводников (М., Изд-во АН СССР, 1963).

[17] M.V. Vedernikov, P.P. Konstantinov, A.T. Burkov. In: Eigth Int. Conf. on Thermoelectric Energy Conversion, ed. by H. Scherer (Nancy, France, 1989) p. 45.

[18] M. Hong, J. Zou, Z.-G. Chen. Adv. Mater., 31 (14), 1807023 (2019).

[19] Л.В. Прокофьева, Ю.И. Равич, П.П. Константинов, А.А. Шабалдин. ФТП, 46, 889 (2012).

[20] X. Zhang, J. Li, X. Wang, Z. Chen, J. Mao, Y. Chen, Y. Pei. J. Am. Chem. Soc., 140, 15883 (2018).

[21] Т.Б. Жукова, Ю.А. Логачев, Л.М. Сысоева. ФТТ, 20, 280 (1978).

[22] J. Goldak, C.S. Barrett. J. Chem. Phys., 44, 3323 (1966).

[23] U.D. Wdowik, K. Parlinski, S. Rols, T. Chatterji. Phys. Rev. B, 89, 224306 (2014).

[24] P.B. Pereira, I. Sergueev, S. Gorsse, J. Dadda, E. Muller, R.P. Hermann. Phys. Status Solidi B, 250 (7), 1300 (2012).

[25] A.T. Burkov, A. Heinrich, P.P. Konstantinov, T. Nakama, K. Yagasaki. Meas. Sci. Technol., 12, 264 (2001).

[26] А.Т. Бурков, А.И. Федотов, А.А. Касьянов, Р.И. Пантелеев, Т. Накама. Науч.-техн. вестн. информационных технологий, механики и оптики, 15, 173 (2015).

Редактор Г.А. Оганесян

\section{Effect of thermal history on the properties of efficient thermoelectric alloys $\mathbf{G e}_{0.86} \mathbf{P b}_{0.1} \mathbf{B i}_{0.04} \mathbf{T e}$}

A.A. Shabaldin ${ }^{1}$ A.Yu Samunin ${ }^{1}$ P.P. Konstantinov ${ }^{1}$ S.V. Novikov ${ }^{1}$, A.T. Burkov ${ }^{1}$, Zhonglin $\mathrm{Bu}^{2}$, Yanzhong $\mathrm{Pei}^{2}$

${ }^{1}$ loffe Institute, 194021 St. Petersburg, Russia

${ }^{2}$ Interdisciplinary Materials Research Center, School of Materials Science and Engineering, Tongji University, Shanghai, China

Abstract In this work, the properties of GeTe-based alloys, doped with bithmus: $\mathrm{Ge}_{0.86} \mathrm{~Pb}_{0.1} \mathrm{Bi}_{0.04} \mathrm{Te}$ were investigated. The aim of the study is to explore the possibility of increasing the thermoelectric efficiency of a compound by combining optimal doping and isovalent substitution to improve the electronic properties with a simultaneous decrease of the lattice thermal conductivity. We studied alloy samples prepared in two different research laboratories using similar, but not completely identical procedures. It is shown that the electronic (thermoelectric power and electrical conductivity) properties of the samples of the two groups are in good agreement with each other. The properties of alloys depend on the thermal history of the samples due to the presence at temperatures of $600-800 \mathrm{~K}$ of a phase transition from a lowtemperature rhombohedral to a high-temperature cubic structural modification. The thermoelectric figure of merit of alloys reaches a maximum value of 1.5 at a temperature of about $750 \mathrm{~K}$. 